\title{
¿POR QUÉ COMUNICARSE EN FORMACIÓN A DISTANCIA?
}

\author{
Cathia Papi, Gustavo Angulo Mendoza, Caroline Brassard, Jean-luc Bédard, Christine Sarpentier \\ Université Téluq, Canadá
}

\begin{tabular}{l} 
PALABRAS CLAVE \\
\hline Comunidad \\
Formación a distancia \\
Interacción
\end{tabular}

\section{RESUMEN}

La formación a distancia (FAD) parece una manera de responder a las exigencias de cualificación de las sociedades modernas favoreciendo el aprendizaje en todo momento de la vida y en cualquier lugar. Sin embargo, este modo de formación sufre a menudo de importantes tasas de abandono causadas en parte por el aislamiento de los estudiantes. El desarrollo de diferentes herramientas de comunicación permite ofrecer a los estudiantes a distancia espacios para interactuar y trabajar juntos. ¿En qué medida los estudiantes aprovechan los dispositivos de interacción propuestos para comunicarse y aprender con los demás? Basado en una revisión sistemática de los estudios publicados durante los diez últimos años sobre estos tipos de dispositivos establecidos en Canadá, nuestro meta-análisis pone en relieve que, de manera general, los estudiantes prefieren aprovechar la flexibilidad de la FAD y trabajar solos. Así, en la mayoría de los dispositivos no son muchos los que comunican. Sin embargo, veremos que, cuando no se trata solamente de socializar sino también de reflexionar sobre una práctica, las interacciones se multiplican.

\section{KEY WORDS}

Community

Distance Education

Interaction

\section{ABSTRACT}

Distance Education (DE) seems a way to meet the qualification requirements of contemporary societies, promoting lifelong learning anywhere. However, this mode of education often suffers from high dropout rates, notably caused by students' isolation. The development of various communication tools offers spaces to interact and work together. To what extent do students take advantage of the proposed interaction devices to communicate and learn with others? Based on a systematic review of studies published over the last ten years addressing these types of devices established in Canada, our meta-analysis highlights that in general, students prefer to take advantage of the flexibility of DE and work alone. Thus, in most devices, there are not many students communicating. However, we will see that, when it is not only about socializing but also reflecting on a practice, interactions are much more frequent. 


\section{Introducción}

$\mathrm{L}$ a formación de las personas cumple un papel crucial en el siglo XXI permitiendo el desarrollo de competencias y la adaptación a las necesidades de las organizaciones. De hecho, la acelerada evolución socioeconómica incita a los individuos a formarse durante toda la vida para adaptarse a las cambiantes necesidades profesionales y para innovar con el fin de crear la sociedad del mañana.

Con las limitaciones impuestas por las actividades profesionales y personales, los adultos a menudo no tienen la oportunidad de tomar cursos presenciales y por ello son cada vez más propensos a recurrir a la formación a distancia. La democratización de Internet desde la década de 1990 y su rápido desarrollo ${ }^{1}$ contribuyeron al crecimiento de la educación a distancia y a su evolución. Lejos de la formación por correspondencia con sus grandes manuales impresos y pocas oportunidades para la comunicación postal o telefónica con los instructores, la formación en línea está ahora al alcance de la mayoría, con recursos multimedia y posibilidades de comunicación accesible en todo momento a través de diferentes pantallas (computadores, tabletas, teléfonos inteligentes) presentes en la vida de todos.

Durante mucho tiempo la FAD fue considerada como un posible paliativo reservado para las personas localizadas en lugares alejados de los establecimientos educativos o para aquellas que deseaban perfeccionarse o incluso retomar los estudios a la edad adulta. En la actualidad, la FAD tiende a percibirse como un modo de formación entre otros, cuya calidad ya no parece ponerse en duda. Ella atrae a un público cada vez más amplio y joven como lo señala Lamy (2011) respecto de los estudiantes de la Open University y Audet (2011) con relación a la evolución de la FAD en Quebec. Sin embargo, la proliferación de cursos y la diversificación de recursos tecnopedagógicos no son suficientes para asegurar el aprendizaje y la perseverancia de los estudiantes hasta el final de su formación. De ahí el constante desarrollo de la investigación en esta área.

\section{Problemática}

Caracterizada por su flexibilidad, la FAD contribuye al aumento del acceso a la educación superior. Si bien esta modalidad de formación surge así como una interesante forma de desarrollo personal o profesional, ella no está exenta de dificultades como lo muestran las altas tasas de deserción. La

\footnotetext{
1 Entre 2000 y 2015, la tasa de penetración de Internet se multiplicó siete veces, pasando de 6,5\% a 43\% de la población mundial. (IUT, 2015 http://www.itu.int/net/pressoffice/press_releases/2015/17fr.aspx\#.VZOrLOdV4mX)
}

persistencia y el aprendizaje a profundidad parecen particularmente más problemáticos, de tal forma que los estudiantes difícilmente se benefician de una dinámica colectiva. Así, para promover el éxito de los estudiantes, se aplican diferentes mecanismos de apoyo al aprendizaje. Algunos han demostrado su eficacia y parecen esenciales, como la tutoría individual o grupal ejercida por una persona remunerada por esta labor (Glikman, 2002; Denis, 2003; Ndoye, 2005; Jacquinot-Delaunay, 2008; Depover et al, 2011). Sin embargo, esta fórmula parece necesaria pero no suficiente, sobre todo en el sentido de que algunos estudiantes no se atreven a entrar en contacto con su tutor o no obtienen respuestas lo suficientemente rápidas u oportunas por parte de este (Loisier, 2010). Además, esta fórmula resulta costosa y como consecuencia de ello el tiempo que un tutor puede dedicar a cada estudiante es relativamente limitado.

Por esta razón se ofrecen ocasionalmente otros tipos de fórmulas complementarias que pueden ayudar a tejer vínculos sociales entre los estudiantes y al mismo tiempo contribuir a la formación. Estas fórmulas se basan en el trabajo entre pares y en el uso de tecnologías de la información y la comunicación (TIC). Cada institución implementa de múltiples maneras un tipo de dispositivo de interacción y de acompañamiento por pares. El principio consiste en la tutoría de un nuevo estudiante por uno antiguo, o en la posibilidad de un sistema de comunicación y ayuda mutua entre los estudiantes que siguen un mismo programa (Deschênes et al, 2003; Papi, 2013). Este último es a veces simplemente sugerido a través del fomento a la interacción y la provisión de herramientas de comunicación sincrónica y asincrónica. Otras veces se incluye en el itinerario de formación a través de situaciones de aprendizaje cooperativo o colaborativo (Dillenbourg, 1999; Henri y Lundgren-Cayrol, 2001; Berlanga et al, 2009; Webb, 2013).

Pero mientras que el alcance potencial del intercambio entre estudiantes parece prometedor, cualquier comunicación o realización colectiva impuesta, así como cualquier uso prescrito de un dispositivo, parecen contravenir la flexibilidad característica de la formación a distancia. Además, la sociología de los usos siempre ha puesto de manifiesto la discrepancia entre el uso de un objeto conforme a lo previsto por sus diseñadores y las prácticas efectivas empleadas (Akrich, 1998). Asimismo, De Certeau (1990) distingue el mundo de la producción del mundo de los usos, siendo este último igualmente creativo debido a los "bricolajes" presentes en la invención cotidiana. Igualmente, se ha señalado que la no utilización de una tecnología o dispositivo no se justifica tanto por la falta de equipos o de habilidades que por las decisiones racionales que conducen a la no utilización (Kellner et al, 2010; Papi, 2012). 
En consecuencia, cabe preguntarse: en cuanto al diseño y los usos de los dispositivos de interacción en FAD, ¿qué ocurre con la adecuación de los objetivos que justifican dicha interacción?

Para responder a esta pregunta, se presentan a continuación los resultados de una revisión de la literatura sobre los dispositivos canadienses de FAD que involucran la interacción mediada por tecnología entre los estudiantes.

\section{Metodología}

Con el fin de limitar las divergencias propias de las diferencias culturales o en relación con el nivel de educación o el tipo de formación, hemos limitado nuestro estudio a la revisión de los trabajos que portan sobre los dispositivos de formación a distancia que ofrecen modalidades de interacción y que tienen lugar en instituciones de educación superior de Canadá. Debido al desarrollo de la web llamada "social" a partir de los años 2004 y 2005, nos hemos interesado en las investigaciones que han sido objeto de publicaciones en actas de congresos o revistas científicas, entre enero de 2005 y diciembre de 2014.

Luego de una búsqueda exploratoria inicial en Google Scholar, fueron consultados los siguientes motores de búsqueda y bases de datos: Academic Search Complete, Cairn, Education Research Complete, Education Source, Eric, Erudit, Francis, Metalib, Open Edition Freemium, Repère (SDM), Science direct, Taylor and Francis, Hal.Archivesouvertes et Wiley Online Library (Wiley Blackwell). También fueron consultados los sitios web de organizaciones especializadas que difunden los resultados de investigaciones en el campo, así como los sitios de las revistas del área.

Se utilizaron principalmente los equivalentes en francés e inglés de las siguientes palabras clave: ambiente personal de aprendizaje, aprendizaje colaborativo, apoyo, aprendizaje colaborativo asistido por ordenador, aprendizaje cooperativo, aula virtual, comunidad, comunidad de aprendizaje, comunidad virtual, comunicación mediada por ordenador, cooperación, dispositivo de aprendizaje mediado por tecnología, educación postsecundaria, educación superior, formación a distancia, formación en línea, interacción, Internet, pares, tutoría y universidad.

Después de leer los resúmenes y en algunos casos los textos completos, se retuvieron inicialmente 80 publicaciones (44 en francés y 36 en inglés). Tras un primer análisis, 20 de ellas fueron excluidas, bien porque abordaban temas conexos al objeto de estudio o porque eran duplicados (por ejemplo, un mismo artículo publicado en inglés y en francés). Así entonces, 60 publicaciones (34 en francés y 26 en inglés) fueron finalmente estudiadas utilizando para ello una misma rejilla de análisis.

Además de los datos de identificación de cada publicación, la rejilla de análisis permitió registrar la(s) categoría(s) de interacción privilegiada(s) por el dispositivo descrito (socialización y/o aprendizaje). Asimismo, se identificaron diferentes elementos que permitieron clasificar las experiencias estudiadas dentro de una tipología: origen del dispositivo, actores, tecnología(s) empleada(s), pedagogía puesta en escena (socioconstructivismo, sociocognitivismo o conectivismo), situaciones de aprendizaje y actividades desarrolladas. Se determinaron también las incidencias del dispositivo analizado, estableciendo la(s) dimensión(es) de apoyo al aprendizaje que querían reforzarse (cognitiva, social, afectiva, motivacional, metacognitiva), así como los impactos positivos y las limitaciones.

\section{Resultados}

El establecimiento de dispositivos de formación basados en la interacción implica la idea de que el conocimiento no es el resultado de la asimilación de un saber externo, sino una construcción intelectual y social. El análisis de las publicaciones seleccionadas muestra que la gran mayoría de los dispositivos se basan en el socioconstructivismo, mientras sólo cuatro estudios hacen referencia al sociocognitivismo $\mathrm{y}$ dos al conectivismo. Los dispositivos presentados involucran elementos de la Web 1.0 (cursos, actividades educativas, videos), así como también otros que se derivan de la Web 2.0 y que se basan en diferentes herramientas de comunicación asincrónica (principalmente foros en 20 publicaciones y mensajes de correo electrónico en nueve publicaciones), comunicación sincrónica (especialmente videoconferencias en diez publicaciones y chats en cinco de ellas; rara vez los microblogs y los SMS) y escritura colaborativa (principalmente el wiki en ocho publicaciones y otras herramientas como los blogs).

Varios dispositivos ofrecen plataformas - Elgg la más citada - que integran diferentes herramientas. Con frecuencia, estas son diseñadas principalmente para fines educativos, como los campus virtuales, los entornos personales de aprendizaje (PLE por su sigla en inglés), los portafolios digitales o incluso los entornos basados en realidad virtual o 3D. Más allá de la uniformidad en la orientación pedagógica y de la gran variedad de herramientas, la compilación de las 60 rejillas de análisis permitió poner en evidencia cuatro tipos de objetivos principales que explican el fomento de la interacción en formación a distancia así como tres tipos de objetivos que llevan a los estudiantes a interactuar.

\section{¿Por qué fomentar la interacción?}

La implementación de dispositivos que ofrecen situaciones de interacción se produce como respuesta a diferentes objetivos: reducir de la distancia, enriquecer la experiencia de aprendizaje, romper el aislamiento y desarrollar competencias. 
Mientras que los dos primeros objetivos se centran en los beneficios de la distancia y en el potencial educativo de la colaboración, ellos sólo fundamentan un pequeño número de dispositivos. Las otras dos familias de objetivos son la fuente de la mayoría de los dispositivos estudiados.

Los dispositivos destinados a reducir la distancia son propios de los contextos en los cuales la única solución parece ser la formación a distancia en colaboración, teniendo en cuenta la dispersión geográfica de las personas para quienes sería apropiado el aprendizaje grupal. En Canadá los francófonos son un grupo minoritario $y$, consecuentemente, son escasos los dispositivos cuyo objetivo sea el de proporcionar posibilidades de establecimiento de redes sociales y colaboración para los francófonos que residen fuera de Quebec (IsaBelle et al, 2010; IsaBelle y Dupláa, 2011). Lo mismo ocurre con la formación continua de los docentes que trabajan en zonas rurales y que se encuentran alejados de sus compañeros o de las instituciones de formación (Pellerin y Allaire, 2010). El sistema de aula virtual ${ }^{2}$ se vislumbra entonces como una posibilidad interesante para ofrecer un mayor número de cursos a estudiantes ubicados en zonas remotas (Allaire et al., 2011).

Los dispositivos concebidos para enriquecer la experiencia se basan en la diversificación de las situaciones de aprendizaje y fomentan particularmente la colaboración como medio de promover el aprendizaje de nociones, conceptos o habilidades. La atención se centra en los escenarios de aprendizaje, tales como los debates en torno a temas del curso (Park et al., 2011), escritura de poesía en un wiki (Dymoke y Hughes, 2009) o seminarios web ${ }^{3}$ (Power y St-Jacques, 2014).

Con frecuencia es puesto en relieve el deseo de romper la sensación de aislamiento de los estudiantes a distancia (De Pauw y Vachon, 2009; IsaBelle et al, 2006; Poellhuber et al, 2012a y b, 2013; Béliveau, 2013; Racette et al, 2014) fomentando el apoyo mutuo y el intercambio (Poellhuber y Chomienne, 2006; Grosjean, 2005; De Pauw y Vachon, 2009, Allaire et al, 2010; IsaBelle y Dupláa, 2011; Poellhuber et al, 2012), así como el establecimiento de una presencia social (Anderson, 2005; IsaBelle, Vézina y Fournier, 2006; Béliveau, 2011; Poellhuber et al, 2012; Chirchi y Landry, 2012; Béliveau, 2013; Racette et al, 2014) y de un sentimiento de cercanía (IsaBelle et al, 2006; Racette y Poellhuber, 2012). Se trata de este modo de contrarrestar la deserción y favorecer la perseverancia en los estudios (Anderson, 2005;

\footnotetext{
2 Como lo señala Wallet (2012), el término “aula virtual" es polisémico y polimórfico. Aquí hacemos referencia a la traducción del término en inglés Virtual Classroom, un sistema que permite reunir en tiempo real a través de Internet a estudiantes y a docentes quienes pueden, principalmente, discutir, verse, leer documentos, ver vídeos, realizar exámenes breves, compartir su pantalla (Gérin-Lajoie et Potvin, 2011).

3 webinars en inglés
}

Poellhuber y Chomienne, 2006, 2009; IsaBelle et al, 2006; Béliveau, 2011; Poellhuber, Racette y Chirchi 2012; Chirchi, Landry, 2012; Béliveau, 2013; Poellhuber et al, 2013; Racette et al, 2014). A menudo se propone la videoconferencia como estrategia para permitir la interacción espontánea en el marco de actividades de participación voluntaria.

Algunos dispositivos proponen la interacción como una forma de garantizar que los estudiantes que se integrarán posteriormente a su oficio como trabajadores o que se encuentran ya en funciones, desarrollen las competencias necesarias para el ejercicio de su profesión. Se trata entonces de enseñarles a funcionar como un equipo, de mostrarles los beneficios de la colaboración y de desarrollar una postura reflexiva. La web social y otras tecnologías son empleadas para promover el aprendizaje favoreciendo la colaboración. En ese sentido, el foro se muestra como la herramienta más utilizada para estimular la interacción en casos específicos ya que democratiza las discusiones, sirve de apoyo a las actividades de aprendizaje en las que se requiere una producción en equipo y exige de los participantes una reflexión profunda sobre el contenido de sus mensajes, para organizarlos y argumentar sus afirmaciones (Nault, 2008). El intercambio se lleva a cabo en torno a los aprendizajes logrados. La actividad de grupo que se propone favorece entonces la adopción de una postura reflexiva sobre la práctica y el desarrollo de habilidades de colaboración dentro del marco mismo del ejercicio de la profesión, tal como sucedería en una pasantía. En esta categoría pueden incluirse los numerosos trabajos sobre la formación de docentes (Allaire, 2008 y 2011; Allaire et al, 2010; Dupláa y Talaat, 2001; Priego y Tomasini, 2014; Rideout et al, 2007), los cursos de producción multimedia a nivel de maestría (Grosjean, 2005) o los estudios sobre la formación de estudiantes en ciencias de la salud (Casimiro et al., 2007).

Tratándose de tipos ideales, múltiples figuras situadas en la intersección de los principales tipos aquí considerados, así como las referencias citadas, pudieran configurarse en forma diferente según los aspectos que han sido tomados en cuenta.

Sin embargo, es interesante hacer notar que en los dispositivos destinados a apoyar la interacción como estrategia para reducir la sensación de aislamiento se señala con frecuencia la dificultad para poner en práctica la colaboración en cursos autorregulados. Así pues, parece que la mayoría de los estudiantes no participan en estas actividades y prefieren trabajar individualmente. Con todo, los pocos estudiantes que intervienen en estos dispositivos parecen obtener un beneficio real de ello, ya que son más persistentes y tienen frecuentemente un mejor desempeño, aunque otros factores pueden contribuir a explicar estos resultados. 
Por el contrario, la renuencia a la colaboración no se menciona en los dispositivos destinados al desarrollo de competencias profesionales. El intercambio de ideas y reflexiones prácticas en el marco de situaciones auténticas parecen, en efecto, ser apreciados por los estudiantes. Sin embargo, por lo general no se indica si hay repercusiones patentes en términos de desempeño académico. Por tanto, debe tenerse en cuenta que no sólo son variables los motivos para la implementación de estos dispositivos de interacción entre estudiantes, sino también lo son las reacciones de estos últimos. Si bien las situaciones de interacción que tienen como objetivo el desarrollo de habilidades profesionales parecen más favorables a la participación comprometida de los estudiantes, las reacciones de estos no son unánimes y requieren ser estudiadas más a fondo.

\section{¿Por qué interactuar?}

Mientras que desarrollan sus estudios a distancia para beneficiarse de una mayor flexibilidad, los usos que los estudiantes hacen de las situaciones de interacción que les son propuestas dependen de las representaciones que ellos tienen de la interacción en la formación. Tres tipos de relación con estas situaciones pueden ser identificados teniendo en cuenta si la interacción les parece irrelevante, si consideran que permite la familiarización con el modo de formación o si piensan que favorece la profundización del aprendizaje y la consolidación de lazos sociales.

Algunos estudiantes no consideran interesante el intercambio con sus pares en el marco de la formación y mantienen de esta manera una relación de indiferencia frente a las situaciones de interacción que le son propuestas (Anderson, Poellhuber y McKerlich, 2010). Puede tratarse bien de estudiantes que están habituados a la FAD en razón de su nivel educativo o de experiencias previas, o bien de personas con poca disponibilidad debido a sus obligaciones familiares o laborales, y que por tales motivos limitan el tiempo dedicado a la formación. La interacción parece carecer de relevancia en relación a su contexto personal de formación, por ello tienden a reducir su participación al mínimo requerido. Estos estudiantes suelen ser muy independientes. Ellos conciben el aprendizaje como una empresa individual y su fuente de conocimiento está en el contenido del curso y en las explicaciones del docente (Anderson, 2005; Poellhuber y Chomienne, 2006, 2009; Chomienne y Marceau, 2009; Chirchi y Landry, 2012). Ellos no están interesados en el trabajo colaborativo (Béliveau, 2011; Racette y Poellhuber, 2012) ni en los contactos sociales al interior de los cursos (Racette et al, 2014). La falta de disponibilidad y de interés en la interacción dificulta el desarrollo de estrategias de aprendizaje en grupo con los estudiantes de este tipo (IsaBelle et al., 2006). Incluso se puede cuestionar la pertinencia de las propuestas de interacción destinadas a estos estudiantes ya que no están muy interesados en ello.

Otros estudiantes, que con frecuencia se encuentran iniciando su proyecto de estudios o que no tienen experiencia alguna en formación en línea, tienen, por el contrario, una relación de exploración frente a las situaciones de interacción que se le proponen. Ellos ven la interacción con sus pares como una forma de familiarizarse con este modo de formación y con sus peculiaridades en términos de itinerarios educativos y de manipulaciones tecnológicas. Estos estudiantes frecuentan los espacios de interacción en búsqueda de información que pueda resultarles útil, así como de compañeros de estudio con quienes compartir recursos, contar cuando requieren ayuda y con quienes puedan trabajar juntos. La participación de estos estudiantes es más bien moderada (Dymoke et Hughes, 2009). Aunque ellos no publican demasiados mensajes, son lectores habituales de las contribuciones de los demás. No obstante, la tasa de publicación puede aumentar en la medida en que comienzan a sentirse cómodos con la tecnología y con las particularidades de la formación en línea. Ellos hacen preguntas a sus compañeros relacionadas con el ambiente de aprendizaje, así como con la propia asignatura. También leen las respuestas y los comentarios de sus pares a las preguntas de otros estudiantes. Los espacios de interacción libre y los foros se convierten para ellos en una importante fuente de apoyo y por tal motivo son consultados con frecuencia (IsaBelle et al., 2006; Racette y Poellhuber, 2012). Estos estudiantes son más propensos a trabajar con otros, son flexibles, demuestran respeto por los acuerdos y se adaptan fácilmente a la dinámica del grupo.

Por último está el tipo de estudiantes que se interesan especialmente en el intercambio entre pares y muestran una real implicación en las situaciones de interacción que le son propuestas (Grosjean, 2005; IsaBelle et al., 2006). A menudo están en una etapa intermedia de su proyecto de estudios y es posible que hayan seguido cursos en línea anteriormente. Se trata frecuentemente de estudiantes con muy fuertes habilidades tecnológicas y quieren compartirlas con los demás. Estos estudiantes se sientan cómodos con el hecho de trabajar en colaboración. Para ellos, los cursos ofrecen tanto la oportunidad de profundizar en el aprendizaje como de establecer y fortalecer los lazos sociales (Conrad, 2005, 2008). Han sido identificados tres factores que motivan la interacción en estos estudiantes.

El objetivo del primer tipo de intercambio de mensajes es promover la proyección social y emocional de los participantes a través de los medios de comunicación utilizados (Goertzen y 
Kristjánsson, 2007). Varios factores determinan los efectos potenciales de este tipo de mensajes: la motivación, la familiaridad, las habilidades tecnológicas, el nivel de implicación y el tipo de actividades a realizar. De igual manera, tres categorías de interacciones se pueden observar: una relativa a las comunicaciones que reflejan libertad de expresión, la que agrupa los intercambios que permiten compartir un vínculo social así como las emociones $\mathrm{y}$, finalmente, una que concentra los mensajes que hacen referencia a la cohesión de grupo (Anderson, 2005; IsaBelle et al., 2006; Béliveau, 2011; Poellhuber et al., 2012; Chirchi y Landry, 2012; Béliveau, 2013; Racette et al., 2014).

El objetivo del segundo tipo de interacciones está relacionado con la materia del curso. Estos intercambios de mensajes demuestran el grado de compromiso de los alumnos con el discurso reflexivo y continuo que lleva a la construcción de sentido, a la confirmación del mismo y su reflexión crítica (Bowen, Farmer y Arsenault, 2012; Nault, 2008). Este tipo de mensajes revela el nivel de desarrollo del pensamiento crítico, considerado, a la vez, como un proceso y como un resultado fundamental de la educación universitaria. Podemos distinguir cuatro categorías de mensajes. La primera reúne las discusiones sobre la identificación de un problema y el cuestionamiento. La segunda categoría corresponde al intercambio de ideas, a los debates y a la exploración de posibles respuestas. Los mensajes más elaborados destinados a la creación de soluciones al problema identificado y a la reflexión sobre el contenido, pertenecen a la tercera categoría. La última categoría de mensajes, indicadora de un aprendizaje de nivel superior, incluye las discusiones sobre las posibles aplicaciones del conocimiento alcanzado.

El tercer tipo de interacción busca la organización del proceso cognitivo con el fin de producir resultados de aprendizaje significativos. En otras palabras, estos mensajes tienen por objeto la gestión de los esfuerzos individuales y colectivos para garantizar así el éxito del trabajo colaborativo (Power y St-Jacques, 2014). Estas interacciones son escasas y a menudo son iniciadas por un miembro del equipo que toma en sus manos la dirección de la actividad del grupo. Aunque la facilitación es una responsabilidad del docente, es importante hacer notar que, en el trabajo colaborativo, los equipos más exitosos tienen algún grado de organización interna. La categorización de este tipo de mensajes ha sido más compleja que para los tipos descritos anteriormente. Sin embargo, observamos ciertos mensajes destinados a planificar el trabajo y otros que revelan el ejercicio de un cierto liderazgo intelectual así como el intercambio de habilidades previamente adquiridas.

En conclusión, la frecuencia de participación es muy alta y variada en los estudiantes que mantienen una relación de implicación frente a la interacción. Ellos demuestran altruismo y encuentran placer al ayudar a sus pares. Estos estudiantes comprometen los esfuerzos que requieren las actividades de grupo y están dispuestos a trabajar por un objetivo común. Su compromiso parece constante. Si bien son pocos, estos estudiantes son un público ideal para los proyectos de aprendizaje colaborativo. Sin embargo, no es raro que estos estudiantes se sientan decepcionados o frustrados (Capdeferro y Romero, 2012). En efecto, las dificultades asociadas con la comunicación, la organización del grupo, la divergencia de objetivos de aprendizaje, la disparidad en los niveles de compromiso de los participantes, la calidad de las contribuciones individuales y la diferencia entre los resultados individuales y colectivos son fuentes de dificultad y de decepción.

\section{Discusión}

Al comparar las razones que guían la implementación de dispositivos que fomentan la interacción con las motivaciones que llevan a los estudiantes a interactuar o no, nos damos cuenta que si bien el aislamiento puede ser un factor que incide negativamente en la perseverancia en los estudios, los estudiantes no lo entienden de la misma manera. Así entonces, mientras que los estudiantes con más ocupaciones o aquellos menos habituados a la formación a distancia serían los mayores beneficiados con la interacción, estos a menudo no buscan entrar en comunicación para de esta forma crear un ambiente que favorezca su proyecto de formación. En consecuencia, estos estudiantes acentúan el riesgo de abandonar los estudios cuando se encuentren con dificultades de cualquier nivel (organizacional, tecnológico, educativo). Aunque algunos estudiantes no trabajan en colaboración debido a su grado de autonomía y a su capacidad de formarse con los recursos propuestos sin apoyo humano adicional, es importante notar que la mayoría de los estudiantes implicados en las interacciones son los que mejor se habitúan a este tipo de formación, al punto de poder percibir lo que les aporta este tipo de intercambios.

Es interesante observar que cuando los estudiantes interactúan, las motivaciones o los tipos de interacción que se perciben corresponden en cierta medida a los elementos del modelo de Community of Inquiry (Col). Este marco de referencia para la instauración de una comunidad ha sido definido por Garrison, Anderson y Archer (2000) y se basa en el aprendizaje a través de la resolución de problemas. El modelo implica una presencia social que corresponde a la capacidad de los participantes para proyectarse social y emocionalmente en la comunidad; una presencia cognitiva que designa el proceso de creación de significado a través de un diálogo continuo y del trabajo en grupo; y una presencia educativa que hace referencia a la facilitación y a la organización 
del trabajo. Este enfoque teórico es de lejos el más referenciado en los estudios analizados, por lo que es natural verlo reaparecer en las interacciones identificadas (Akyol y Garrison, 2008; Anderson et al., 2010; Archibald, 2012; Conrad, 2005; Goertzen y Kristjánsson, 2007; Jahng, Nielsen y Chan, 2010; Lord y Lomicka, 2014; Munich, 2014; Mykota y Duncan, 2007; Park, Kier y Jugdev, 2011; Power y St-Jacques, 2014; Saint-Jacques, 2013; Vaughan, 2012). En efecto, hemos podido identificar un primer tipo de interacciones que contribuyen a la creación de la presencia social, fomentando la comunicación abierta entre los participantes; un segundo tipo que favorece la presencia cognitiva a través de la externalización de la reflexión de los estudiantes y un tercer tipo que reúne las estrategias implementadas para cumplir los objetivos de aprendizaje conforme a la idea de la presencia educativa.

La presencia educativa se inscribe en el diseño del curso. Esta orienta y facilita el logro de los objetivos educativos modelando un diálogo constructivo. Mientras que la presencia social parece necesaria para el surgimiento tanto de la presencia educativa como de la presencia cognitiva, es importante insistir en la necesidad, en primer lugar, de explicar el interés de los estudiantes por la interacción (ya que no resulta algo obvio para la mayoría de ellos) y, en segundo lugar, de crear situaciones de aprendizaje auténticas. Aunque resulten muy exigentes, sólo estas últimas parecen poder llevar al conjunto de estudiantes a hacer un esfuerzo para interactuar y, con ello, a crear lazos sociales de los cuales podrán beneficiarse una vez terminada la formación. Mientras que algunos estudiantes necesitan estos intercambios para familiarizarse con este modo de formación, podríamos recomendar que, cuando resulte pertinente, se prevea una situación de aprendizaje colaborativo al inicio de la formación. De esta manera se establecerían al mismo tiempo los lazos sociales que podrían promover la creación de una comunidad e inducir las nuevas dinámicas de formación.

\section{Agradecimientos}

Los autores agradecen al Gobierno de Canadá que financió el presente estudio por intermedio del Consejo de investigaciones en ciencias humanas (Conseil de recherches en sciences humaines, CRSH. Página web: http://www.sshrc-crsh.gc.ca/). 


\section{Referencias}

Akyol, Z. y Garrison, D.R. (2008). The Development of a Community of Inquiry over Time in an Online Course: Understanding the Progression and Integration of Social, Cognitive and Teaching Presence. [Reports Evaluative]. Journal of Asynchronous Learning Networks, 12(3-4), 3-22. Recuperado de http://sloanconsortium.org/jaln/v12n3/ development-community-inquiry-over-time-online-courseunderstanding-progression-and-integ

Akrich, M. (1998). Les utilisateurs, acteurs de l'innovation. Education permanente (134), 79-90.

Allaire, S. (2008). Soutenir le cheminement de stage d'apprentis enseignants au secondaire par un environnement d'apprentissage hybride. Canadian Journal of Learning and Technology, 34(2). Recuperado de http://www.cjlt.ca/index.php/cjlt/article/view/492/ 223

- (2011). Le discours asynchrone d'une communauté d'apprentissage en réseau: source reconnue de l'action en classe par des stagiaires en enseignement. Revue internationale des technologies en pédagogie universitaire/International Journal of Technologies in Higher Education, 8(3), 49-65.

Allaire, S., Pellerin, G., Beaudoin, M., Couture, C. y Turcotte, S. (2010). Développement d'un programme de formation interuniversitaire en réseau: pallier une situation découlant des mouvements démographiques au Québec. Journées Communication et Apprentissage Instrumentés en Réseau (JOCAIR 2010).

Anderson, T. (2005). Distance learning-Social software's killer ap? The Open \& Distance Learning Association of Australia. Australia. Recuperado de http://hdl.handle.net/2149/ 2328

Anderson, T., Poellhuber, B., y McKerlich, R. (2010). Self paced learners meet social software: an exploration of learners' attitudes, expectations and experience. Online Journal of Distance Learning Administration, 13(3).

Archibald, D. (2012). An Online Resource to Foster Cognitive Presence. Educational Communities of Inquiry: Theoretical Framework, Research and Practice: Theoretical Framework, Research and Practice, 168.

Audet, L. (2011). Regards sur l'évolution de la formation à distance au Canada francophone. Distances et savoirs, 9(3), 313-330. Recuperado de http://www.cairn.info/revue-distances-et-savoirs-2011-3-page-313.htm

Béliveau, D. (2011). L'utilisation des logiciels sociaux et de la visioconférence Web pour développer la présence sociale et favoriser la collaboration entre pairs en formation à distance. Montréal : Cégep@distance.

- (2013). Osmose : Bilan d'une expérimentation menée au Cegep@distance. Pédagogie collégiale, 26(2), 35-40.

Berlanga, A., Rusman, E., Bitter-Rijpkema, M. y Sloep, P. (2009). Guidelines to Foster Interction in Online Communities. Dans Koper, R. (dir.), Learning Network Services for Professional Development (p. 27-42). Berlin Heidelberg : Springer-Verlag.

Bowen, G.M., Farmer, R. y Arsenault, N. (2012). Perspectives on the Use of "Anonymous" Discussion Forums in Undergraduate Education Courses. [Reports - Research]. Canadian Journal of Learning and Technology, 38(2), 27.

Casimiro, L., Tremblay, M., Bouchard-Lamothe, D. y Hall, P. (2007). Vers un modèle de collaboration novateur pour l'enseignement interprofessionnel: conception d'un atelier en ligne pour stimuler le travail en milieu rural. Francophonies d'Amérique (23-24), 45-66.

Chirchi, M. y Landry, C. (2011). Projet Osmose du Cegep à distance : collaboration "stigmergique" en FAD autorythmée.Cégep@distance. Montréal.

Chomienne, M. y Marceau, F. (2009). Un environnement de réseautage social pour apprendre au Cégep@ distance. Revue internationale des technologies en pédagogie universitaire/International Journal of Technologies in Higher Education, 6(2-3), 63-70.

Conrad, D. (2005). Building and Maintaining Community in Cohort-Based Online Learning. [Reports - Research]. Journal of Distance Education, 20(1), 1-20. Recuperado de http://www.jofde.ca/index.php/jde/article/view/78

- (2008). From Community to Community of Practice: Exploring the Connection of Online Learners to Informal Learning in the Workplace. [Reports - Evaluative]. American Journal of Distance Education, 22(1), 3-23. Recuperado de rmaworld.com/openurl?genre=article\&id=doi:10.1080/08923640701713414

De Certeau, M. (1990). L'invention du quotidien, 1. Arts de faire. Paris : Gallimard.

De Pauw, F. y Vachon, F. (2009). La formation collégiale à distance en français en Colombie-Britannique : une expérience concluante. Pédagogie Collégiale 22(3), 22-28.

Denis, B. (2003). Quels rôles et quelle formation pour les tuteurs intervenant dans des dispositifs de formation à distance? Distances et savoirs, 1(1), 16-24.

Depover, C., De Lièvre, B., Peraya, D., Quintin, J.J. y Jaillet, A. (2011). Le tutorat en formation à distance. Bruxelles : De Boeck.

Deschênes, A.-J., Bégin-Langlois, L., Charlebois-Refae, N., Côté, R. y Rodet, J. (2003). Description d'un système d'encadrement par les pairs et de la formation des pairs anciens. Revue de l'éducation à distance, 18(1), 19-41. 
Dillenbourg, P. (1999). What do you mean by collaborative learning? En Dillenbourg, P. (dir.), Collaborativelearning: Cognitive and computational approaches (p. 1-19). Oxford: Elsevier.

Duplàa, E. y Talaat, N. (2012). Connectivisme et formation en ligne. Distances et savoirs, 9(4), 541-564.

Dymoke, S. y Hughes, J. (2009). Using a Poetry Wiki: How Can the Medium Support Pre-Service Teachers of English in Their Professional Learning about Writing Poetry and Teaching Poetry Writing in a Digital Age? English Teaching: Practice and Critique, 8(3), 91-106.

Garrison, D.R., Anderson, T. y Archer, W. (2000). Critical inquiry in a text-based environment: Computer conferencing in higher education. The internet and higher education, 2(2), 87-105.

Gérin-Lajoie, S. y Potvin, C. (2011). Évolution de la formation à distance dans une université bimodal. Distances et savoir, 9(3), 349-374.

Glikman, V. (2002). Apprenants et tuteurs: une approche européenne des médiations humaines. Education permanente, 3(152), 55-69.

Goertzen, P. y Kristjansson, C. (2007). Interpersonal Dimensions of Community in Graduate Online Learning: Exploring Social Presence through the Lens of Systemic Functional Linguistics. [Reports - Research]. Internet and Higher Education, 10(3), 212-230. Recuperado de http://dx.doi.org/10.1016/j.iheduc.2007.06.005

Grosjean, S. (2005). Communautés Virtuelles et Communication Médiatisée par Ordinateur-Analyse de Formes de" Ritualisation Sociale. Revue d'Interaction Homme-Machine Vol, 6(1).

Henri, F. y Lundgren-Cayrol, K. (2001/2003). Apprentissage collaboratif à distance. Pour comprendre et concevoir les environnements d'apprentissage virtuels. Sainte-Foy: Presses de l'Université du Québec.

IsaBelle, C. y Duplàa, E. (2011). Formation en ligne: types d'interaction souhaités chez des directions d'école et des enseignants franco-canadiens. Revue internationale des technologies en pédagogie universitaire/International Journal of Technologies in Higher Education, 8(3), 36-48.

IsaBelle, C., St-Amant, G.E. y Fournier, H. (2010). Premier référentiel des connaissances associées aux compétences individuelles et aux capacités organisationnelles du travail collaboratif soutenu par les TIC dans un contexte de formation à distance.

IsaBelle, C., Vézina, N. y Fournier, H. (2006). Un environnement 3D qui favorise le sentiment d'appartenance en situation de formation à distance. Canadian Journal of Learning and Technology/La revue canadienne de l'apprentissage et de la technologie, 32(2).

Jacquinot-Delaunay, G. (2008). Accompagner les apprentissages : le tutorat « pièce maîtresse et parent pauvre » des dispositifs de formation médiatisés. Dans Jacquinot-Delaunay, G. et Fichez, E. (dir.), L'université et les TIC. Chronique d'une innovation annoncée (p. 179-222). Bruxelles : De Boeck.

Jahng, N., Nielsen, W.S. y Chan, E. K. (2010). Collaborative Learning in an Online Course: A Comparison of Communication Patterns in Small and Whole Group Activities. [Reports - Research]. Journal of Distance Education, 24(2), 39-58.

Kellner, C., Massou, L. y Morelli, P. (2010). (Re)penser le non-usage des TIC. Questions de Communication(18), 7-20.

Lamy, M.-N. (2011). Où va la distance, par des temps agités ? Réponses d'une organisation distancielle, l'Open University. Distances et savoirs, 9(4), 611-618. Recuperado de http://www.cairn.info/revue-distanceset-savoirs-2011-4-page-611.htm

Loisier, J. (2010). Mémoire sur l'encadrement des étudiant(e)s dans les formations en ligne offertes aux différents niveaux d'enseignement. Document préparé pour le Réseau d'enseignement francophone à distance du Canada

Lord, G. y Lomicka, L. (2014). Twitter as a Tool to Promote Community among Language Teachers. [Reports Research]. Journal of Technology and Teacher Education, 22(2), 187-212. Recuperado de http://www.editlib.org/p/49768/

Munich, K. (2014). Social Support for Online Learning: Perspectives of Nursing Students. International Journal of E-Learning \& Distance Education, 29. Recuperado de http://ijed e.ca/index.php/jde/article/view/891

Mykota, D. y Duncan, R. (2007). Learner Characteristics as Predictors of Online Social Presence. [Reports Research]. Canadian Journal of Education, 30(1), 157-170. Recuperado de http://www.csse.ca/CJE/Articles/CJE30-1.html

Nault, G. (2008). Le forum de discussion : réflexion sur son potentiel collaboratif en enseignement supérieur. Pédagogie collégiale, 21(4), 16-19.

Ndoye, A.K. (2005). Les rôles du tuteur dans la formation à distance des professeurs vacataires sénégalais. DistanceS (8). Recuperado de http://cqfd.teluq.uquebec.ca/distances/v8n1_e.html

Papi, C. (2012). Causes et motifs du non-usage de ressources numériques. Recherches \& éducations (6), 127-142. Recuperado de http://rechercheseducations.revues.org/1074

- (2013). Le tutorat de pairs dans l'enseignement supérieur: enjeux institutionnels, technopédagogiques, psychosociaux et communicationnels. Paris : l'Harmattan.

Park, C., Kier, C. et Jugdev, K. (2011). Debate as a Teaching Strategy in Online Education: A Case Study. [Reports Research]. Canadian Journal of Learning and Technology, 37(3), 17. 
Pellerin, G. y Allaire, S. (2010). Une formation universitaire pour des écoles en réseau. Québec français(159), 6264. Recuperado de http://id.erudit.org/iderudit/61593ac

Poelhuber, B. y Chomienne, M. (2006). L'amélioration de la persévérance dans les cours de formation à distance: les effets de l'encadrement et de la collaboration. : Cégep@ distance.

Poellhuber, B., Anderson, T., Racette, N. y Upton, L. (2013). Distance Students' Readiness for and Interest in Collaboration and Social Media. [Reports - Research]. Interactive Technology and Smart Education, 10(1), 63-78. Recuperado de http://dx.doi.org/10.1108/17415651311326455

Poellhuber, B. y Racette, N. (2013). Le profil technologique des étudiants canadiens en formation à distance et les déterminants de leur intérêt envers la collaboration et l'utilisation des logiciels sociaux. TIC, technologies émergentes et Web 2.0: quels impacts en éducation?, 335.

Poellhuber, B., Racette, N. y Chirchi, M. (2012). De la présence dans la distance par la visioconférence Web. Revue internationale des technologies en pédagogie universitaire/International Journal of Technologies in Higher Education, 9(1-2), 63-77.

Power, M. y St-Jacques, A. (2014). The Graduate Virtual Classroom Webinar: A Collaborative and Constructivist Online Teaching Strategy. Journal of Online Learning and Teaching, 10(4), 681.

Priego, S. y Bassols, L.T. (2014). Le wiki: un outil pertinent pour des projets de tutorat en ligne? Synergies Mexique (4), 121-132.

Racette, N. y Poellhuber, B. (2012). Les outils de collaboration dans les cours à distance, une affaire de contexte. Intégration Technologique et Nouvelles Perspectives d'Usage, 202.

Racette, N., Poellhuber, B. y Fortin, M.-N. (2014). Dans les cours à distance autorythmés: la difficulté de communiquer. Expérimentation d'un logiciel social et d'une visioconférence dans deux cours à distance autorythmés-deuxième itération. Distances et médiations des savoirs. Distance and Mediation of Knowledge (7).

Rideout, G., Bruinsma, R., Hull, J. y Modayil, J. (2007). Online learning management systems (LMS) and sense of community: A pre-service practicum perspective. Canadian Journal of Learning and Technology, 33(3). Recuperado de http://www.cjlt.ca/ index.php/cjlt/article/view/165/161

Saint-Jacques, A. (2012). Effective Teaching Practices to Foster Vibrant Communities of Inquiry in Synchronous Online Learning. Dans Akyol, Z. et Garrison, D. R. (dir.), Educational Communities of Inquiry: Theoretical Framework, Research and Practice: Theoretical Framework, Research and Practice (p. 84-108).

Vaughan, N. (2012). Community of Inquiry Framework, Digital Technologies, and Student Assessment in Higher Education. Dans Akyol, Z. y Garrison, R. D. (dir.), Educational Communities of Inquiry: Theoretical Framework, Research and Practice: Theoretical Framework, Research and Practice (p. 334-348).

Wallet, J. (2012). De la synchronie médiatisé en formation à distance, les classes virtuelles une appellation mal contrôlée. STICEF, 19. Recuperado de http://sticef.univ-lemans.fr/num/vol2012/14rwallet/sticef_2012_wallet_14r.htm

Webb, N.M. (2013). Information processing approaches to collaborative learning. Dans Hmelo-Silver, C. E., Chinn, C. A., Chan, C. K. K. et O’Donnell, A. (dir.), The International Handbook of Collaborative Learning (p. 1940). New York : Routledge. 